\title{
Graft-versus-host Disease Complicated by Sequential Septic Arthritis and Osteomyelitis
}

\begin{abstract}
JOHN B. MILLER (1), MD, Division of Rheumatology, Department of Medicine, Johns Hopkins University School of Medicine; EDWARD F. McCARTHY, MD, Department of Pathology, Johns Hopkins University School of Medicine; ALLAN C. GELBER \, MD, Division of Rheumatology, Department of Medicine, Johns Hopkins University School of Medicine, Baltimore, Maryland, USA. Address correspondence to Dr. A.C. Gelber, Johns Hopkins University School of Medicine, 5200 Eastern Ave., Mason F. Lord Bldg., Center Tower, Suite 4100, Baltimore, Maryland 21224, USA. E-mail: agelber@jhmi.edu. Dr. Miller's effort was supported by the Cupid Foundation. The Office of Human Subjects Research/Institutional Review Board of Johns Hopkins University School of Medicine has granted approval to proceed with this publication. The patient whose clinical course is portrayed has consented to this publication.

J Rheumatol 2020;47:477-8; doi:10.3899/jrheum.181302
\end{abstract}

Concurrent septic arthritis and osteomyelitis present a challenging situation in a medically complex patient with joint and bone pain. We report on an immunosuppressed patient who developed urosepsis with bacteremia followed sequentially by septic arthritis and osteomyelitis, emphasizing the potential musculoskeletal sites of disseminated bacterial infection in the susceptible host.

A 46-year-old woman presented with unilateral knee pain of 3 months' duration. Her history was notable for follicular lymphoma, treated with rituximab (RTX) and cyclophosphamide, doxorubicin, vincristine, and prednisone chemotherapy. Relapse warranted RTX and ifosfamide, carboplatin, and etoposide chemotherapy and allogeneic stem cell transplantation. Thereafter, she developed graft-versus-host disease with severe cutaneous, hepatic, and colonic manifestations, managed with high-dose prednisone, mycophenolate mofetil, and tacrolimus.

She was then hospitalized for urosepsis with Klebsiella aerogenes bacteremia and received 14 days of cefepime, $1 \mathrm{~g}$ thrice daily. Two weeks after completion, she returned with left knee pain, swelling, and fever. Arthrocentesis yielded 86,000 white blood cells $/ \mathrm{mm}^{3}$ ( $>90 \%$ neutrophils); cultures grew $K$. aerogenes. She underwent operative irrigation and drainage for septic arthritis, treated with 6 weeks of cefepime, escalated to $2 \mathrm{~g}$ thrice daily. Noticeable improvement ensued.

Three months later the woman became wheelchair-dependent and felt intolerable pain with any knee movement. Examination showed marked proximal tibial tenderness. Radiographs demonstrated a moth-eaten appearance, raising concerns about infection or metastasis (Figure 1A). Magnetic resonance imaging identified endosteal scalloping, cortical destruction, and bone infarcts (Figure 1B). Histopathology revealed densely packed neutrophils (Figure 2) consistent with acute osteomyelitis; bone tissue grew $K$. aerogenes with unchanged antibiotic sensitivities; lymphoma was absent. She underwent surgical debridement, placement of antibiotic beads, and prolonged antimicrobial therapy with ciprofloxacin.

Concurrent septic arthritis and osteomyelitis are commonly encountered in children, yet seldom in adults ${ }^{1,2,3}$. Arterial anastomoses between metaphyseal and epiphyseal vessels allow communication between nutrient arteries supplying bone and joint structures. Risk factors for concurrent osteomyelitis include prolonged symptoms, bacteremia, and staphylococcal infection ${ }^{3}$. Postoperatively, the patient achieved substantial reduction in pain and resumed independent ambulation.

\section{REFERENCES}

1. Atcheson SG, Ward JR. Acute hematogenous osteomyelitis progressing to septic synovitis and eventual pyarthrosis. The vascular pathway. Arthritis Rheum 1978;21:968-71.

2. Goldenberg DL, Reed JI. Bacterial arthritis. N Engl J Med 1985;312:764-71.

3. Branson J, Vallejo JG, Flores AR, Hulten KG, Mason EO, Kaplan SL, et al. The contemporary microbiology and rates of concomitant osteomyelitis in acute septic arthritis. Pediatr Infect Dis J 2017;36:267-73.

Personal non-commercial use only. The Journal of Rheumatology Copyright @ 2020 . All rights reserved. 


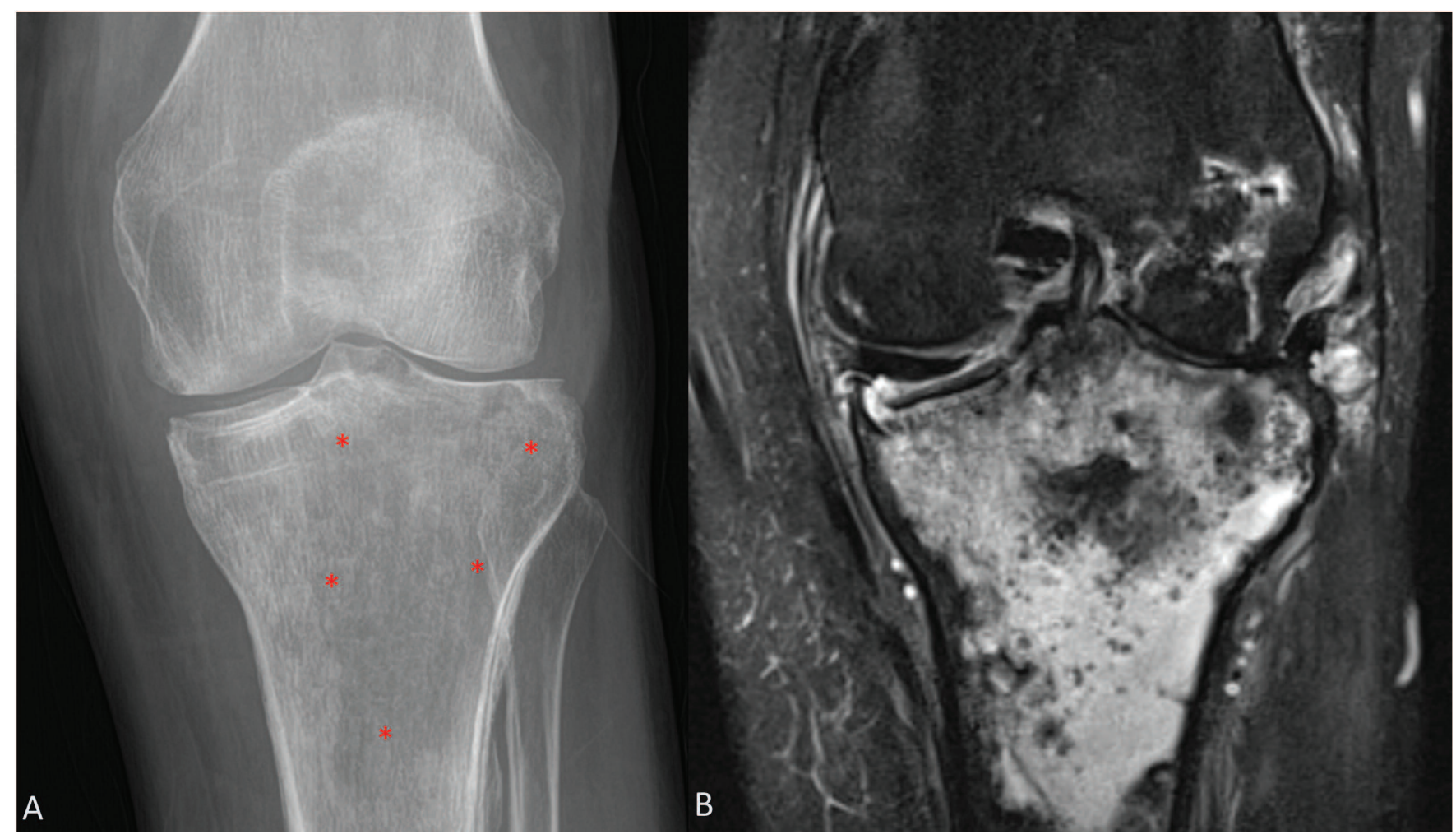

Figure 1. A. Knee radiograph demonstrating diffuse osteopenia affecting the proximal tibia, along with several foci $(*)$ of bone lysis with adjacent sclerotic rims creating a moth-eaten appearance. B. Magnetic resonance imaging of the left knee showing a marrow-replacing lesion involving the proximal tibial metaphysis. There is heterogeneous marrow edema with cortical destruction and soft tissue extension.

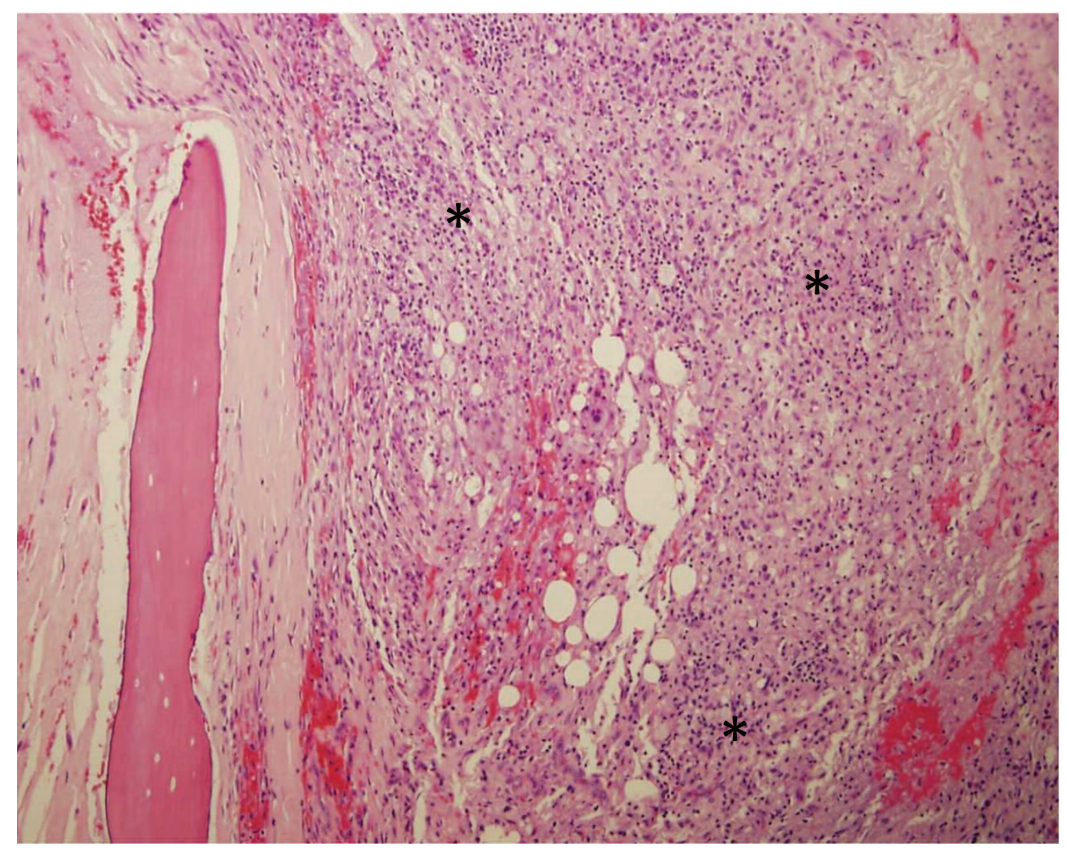

Figure 2. Bone histopathology reveals bone necrosis and surrounding dense neutrophilic infiltration (asterisks) characteristic of acute osteomyelitis. There was no evidence of lymphoma. 